\title{
Hilar Portion of the Hepatic Duct
}

National Cancer Institute

\section{Source}

National Cancer Institute. Hilar Portion of the Hepatic Duct. NCI Thesaurus. Code C43630.

The segment of either hepatic duct located in the hilum of the liver. 